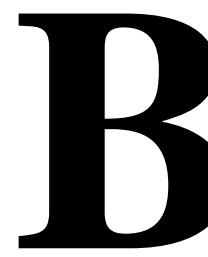

\title{
UDGET AND STRATEGY: BEYOND BUDGETING AS A TOOL FOR PLANNING AND BUDGETARY CONTROL IN A GRADUATION COURSE
}

\author{
1 Thiago Coelho Soares \\ ${ }^{2}$ Jonas Schneider \\ ${ }^{3}$ Carlos Montenegro Lima \\ ${ }^{4}$ Sandro Vieira Soares
}

\begin{abstract}
Objective: In this article we aim to analyze the strategic positioning of a distance education undergraduate course and verify its operationalization through the budget.
\end{abstract}

Method: Documental research and interview were the primary methods utilized. Documents from the university were analyzed, as well as the specific planning developed by the course coordinator.

Originality / Relevance: There is evidence on the relationship between budget planning and control. However, there is a portion to be filled regarding the management of higher education institutions. Therefore, this research applied a recent budget method (Beyond Budgeting) as a way to highlight the relationship between planning and control.

Results: Strategic positioning of course differentiation was identified according to Porter (2004), and Beyond Budgeting was chosen as a tool to aid organizational planning. The operationalization of the strategy through Beyond Budgeting was used to show the relationship between the strategic positioning of the course, the planning of the university and the budget piece elaborated in this research.

Theoretical / methodological contributions: The operationalization of the strategy through Beyond Budgeting highlighted the relationship between the strategic positioning of the course, the planning of the university and the budget piece elaborated in this research.

Keywords: Planning, Budgeting, Beyond Budgeting.

\section{Cite it like this:}

Soares, T., Schneider, J., Lima, C., \& Soares, S. (2019). Orçamento e estratégia: Beyond Budgeting como ferramenta de planejamento e controle orçamentário em um curso de graduação. Revista Ibero-Americana De Estratégia, 18(1). 126-138. https://doi.org/10.5585/ijsm.v18i1.2646

\footnotetext{
${ }^{1}$ Universidade do Sul de Santa Catarina, UNISUL, Santa Catarina, Brasil. Email: thiago.soares@unisul.br Orcid Id: https://orcid.org/0000-0002-7470-6271

${ }^{2}$ Universidade do Sul de Santa Catarina, UNISUL, Santa Catarina, Brasil. Email: jonas.schneider@unisul.br Orcid Id: https://orcid.org/0000-0003-2555-7043

${ }^{3}$ Universidade do Sul de Santa Catarina, UNISUL, Santa Catarina, Brasil. Email: carlos.montenegro@unisul.br

${ }^{4}$ Universidade do Sul de Santa Catarina, UNISUL, Santa Catarina, Brasil. Email: sandrovieirasoares@ hotmail.com Orcid Id: https://orcid.org/0000-0001-7076-4936
} 

e controle orçamentário em um curso de graduação

\section{ORÇAMENTO E ESTRATÉGIA: BEYOND BUDGETING COMO FERRAMENTA DE PLANEJAMENTO E CONTROLE ORÇAMENTÁRIO EM UM CURSO DE GRADUAÇÃO}

\section{Resumo}

Objetivo: Este artigo tecnológico tem como objetivo analisar o posicionamento estratégico de um curso de graduação de ensino a distância e verificar a sua operacionalização por meio do orçamento.

Método: Realizou-se pesquisa documental e entrevista. Foram analisados documentos da universidade, bem como o planejamento específico elaborado para o curso elaborado pelo coordenador do curso.

Originalidade/Relevância: Apesar de existir evidências sobre a relação planejamento e controle orçamentário, há uma coluna a ser preenchida no que tange a gestão de instituições de ensino superior. Portanto esta pesquisa aplicou um método orçamentário recente (Beyond Budgeting) como forma de evidenciar a relação planejamento e controle.

Resultados: Identificou-se o posicionamento estratégico de diferenciação do curso, segundo Porter (2004) e optou-se pelo Beyond Budgeting como ferramenta de auxílio ao planejamento organizacional. A operacionalização da estratégia por meio do Beyond Budgeting foi utilizada para evidenciar a relação existente entre o posicionamento estratégico do curso, o planejamento da universidade e a peça orçamentária elaborada nesta pesquisa.

Contribuições teóricas/metodológicas: A operacionalização da estratégia por meio do Beyond Budgeting foi utilizada para evidenciar a relação existente entre o posicionamento estratégico do curso, o planejamento da universidade e a peça orçamentária elaborada nesta pesquisa.

Palavras-chave: Planejamento, Orçamento, Beyond Budgeting.

\section{PRESUPUESTO Y ESTRATEGIA: BEYOND BUDGETING COMO HERRAMIENTA DE PLANIFICACIÓN Y CONTROL DEL PRESUPUESTO EN UN CURSO DE GRADUACIÓN}

\section{Resumen}

Objetivo: Este artículo tiene como objetivo analizar el posicionamiento estratégico de un curso de graduación de enseñanza a distancia y verificar su funcionamiento a través del presupuesto.

Método: Se realizó la investigación documental y la entrevista. Se analizaron documentos de la universidad, así como la planificación específica elaborada para el curso elaborado por el coordinador del curso.

Originalidad / Relevancia: A pesar de existir evidencias sobre la relación planificación y control presupuestario, hay una columna a ser llenada en lo que se refiere a la gestión de instituciones de enseñanza superior. Por lo tanto, esta investigación aplicó un método presupuestario reciente (Beyond Budgeting) como una forma de evidenciar la relación de planificación y control.

Resultados: Se identificó el posicionamiento estratégico de diferenciación del curso, según Porter (2004) y se optó por el Beyond Budgeting como herramienta de auxilio a la planificación organizacional. El funcionamiento de la estrategia a través del Beyond Budgeting fue utilizada para evidenciar la relación existente entre el posicionamiento estratégico del curso, la planificación de la universidad y la pieza presupuestaria elaborada en esta investigación.

Contribuciones teóricas / metodológicas: El funcionamiento de la estrategia a través del Beyond Budgeting fue utilizada para evidenciar la relación existente entre el posicionamiento estratégico del curso, la planificación de la universidad y la pieza presupuestaria elaborada en esta investigación.

Palabras clave: Planificación, Presupuesto, Beyond Budgeting. 


\section{INTRODUÇÃO}

Os fatores que caracterizam o posicionamento estratégico de uma universidade é um dos aspectos determinantes para a obtenção de vantagem competitiva. Diversos autores vêm procurando enfatizar a relação entre estratégia e orçamento (Simons, 1987; Langfield-Smith, 1997; Guilding, Cravens \& Tayles, 2000; Hansen \& Van der Stede, 2004; Backes, et al, 2004; Almeida, et al, 2009; Neitzke \& Espejo, 2016; Silva \& Rissi; 2017, Melo et al, 2017).

A falta de alinhamento do orçamento com o planejamento estratégico torna o processo orçamentário pouco eficiente no gerenciamento estratégico. Os orçamentos anuais apresentam bons resultados quando as condições de mercado estão estáveis, os competidores são conhecidos e as ações das pessoas são previsíveis. Nessas condições, poucos tomam decisões, os preços acabam refletindo os custos internos, a estratégia e o ciclo de vida dos produtos são longos e os clientes têm poucas opções de escolha. A centralização exagerada na definição das metas orçamentárias inviabiliza a participação e motivação dos colaboradores.

O orçamento é determinado pela alta administração, assim, os orçamentos são baseados na centralização da gestão. Com isso, atrapalham as tentativas de mudança organizacional, como gerenciamento em equipe e empowerment. Porém, o mercado sofreu alterações e tais características mencionadas anteriormente não fazem mais parte do cenário de muitas empresas. Com o ambiente de mercado dinâmico e com o trabalhador do conhecimento, o processo orçamentário centralizado perde sua eficácia, até porque consome muito tempo e dinheiro (Bornia \& Lunkes, 2007).

Conforme Maciel (2007) uma análise da relação entre intenção estratégica e desempenho passa a merecer destaque. Assim, as ferramentas são úteis para as organizações, mas necessitam de adaptação aos tempos modernos. Desta forma, o objetivo do artigo é analisar o posicionamento estratégico de um curso de graduação de ensino a distância e verificar a sua operacionalização por meio do orçamento.

A pesquisa se justifica na medida no ponto de vista teórico, pois o planejamento orçamentário por meio do Beyond Budgeting é pouco explorado na literatura. No ponto de vista prático revela informações importantes para o planejamento e controle por meio de drivers de desempenho. A escolha do tema se baseia em Berland, Curtis e Sponem (2018) que afirmam que os orçamentos tradicionais não conseguiram contribuir para a gestão das tensões associadas à crescente complexidade dos modelos de negócios, assim o Beyond Budgeting surge como opção para o planejamento orçamentário.

\section{REVISÃO DA LITERATURA}

A estratégia consiste em uma posição conduzida por um conjunto específico de atividades alinhadas aos objetivos da organização, que visa a proporcionar valor para o ambiente competitivo (Porter, 2004). Conforme o autor existem três estratégias: liderança em custos, diferenciação e enfoque.

O posicionamento estratégico em Liderança no custo é uma posição baseado no baixo custo, na redução dos custos a uma margem menor que os concorrentes. A Diferenciação consiste em produzir ou oferecer produtos e serviços com valor agregado, considerado único e diferenciado quando comparado com os concorrentes. O Enfoque consiste em focar em um determinado grupo, linha ou mercado no campo de atuação da empresa (Porter, 2004).

Conforme Serra, Torres e Torres (2004), existem três componentes que definem a estratégia: a) Posicionamento: é a criação de uma posição valiosa e única que envolve um conjunto diferente de atividades. Consiste em desempenhar as mesmas atividades de diferentes maneiras das exercidas pelos competidores; b) Opções Excludentes (trade-off): requer que sejam feitas opções para competir, ou seja, deve-se escolher o que não deve ser feito; e c) Sinergia: implica em criar sinergia entre as atividades da organização. As escolhas de posicionamento e os trade-offs determinam não só as atividades que devem ser descartadas, mas também o modo como devem relacionar-se umas com as outras.

As estratégias originam-se no processo de planejamento estratégico bem como nas atividades empresariais. Assim, existe impacto da estratégia no uso do orçamento, ou seja, a estratégia organizacional influencia o estilo de uso do orçamento (Neitzke \& Espejo, 2016).

O orçamento e o planejamento estratégico são instrumentos que auxiliam na gestão das instituições de ensino superior. O orçamento é apoio ao processo 

e controle orçamentário em um curso de graduação

de decisão dos gestores e por essa razão está relacionado à estratégia das instituições. Para serem competitivas e desempenharem o seu papel social, as universidades fazem uso destas duas ferramentas (Silva e Rissi, 2017).

Por meio do planejamento e controle e estabelecendo as capacidades de recursos, os gestores desenvolvem competências para atender os objetivos estratégicos de capacidade, qualidade, tempo e custo, gerenciando de forma mais eficaz. Com a vinculação do orçamento em curto prazo e a estratégia de longo prazo, pode-se garantir a eficácia do planejamento e controle dos fatores críticos de sucesso (Backes, et al, 2004).

As estratégias são diferentes entre si e implicam em diferentes abordagens orçamentárias. Assim, a abordagem orçamentária é dependente da estratégia escolhida e implementada. O orçamento é uma ferramenta que pode ser utilizada tanto no apoio à formulação e implementação das estratégias, como no controle do orçamento (Almeida et al, 2009).

Durante entrevistas em algumas empresas Henttu-Aho e Järvinen (2012) perceberam uma mudança nas práticas cotidianas de orçamento e mudança no significado compartilhado comum de orçamento no discurso das pessoas. O orçamento mestre tradicional como um documento formal e processo de longa data foi fragmentado como uma coleção de várias ferramentas, tais como orçamento de custos fixos, definição de metas, o plano anual de Benchmarking, Balanced Scorecard e Gestão Baseada em Valor. Nesta prática o orçamento recémformulado pode, no entanto, identificar as principais funções do orçamento, tais como planejamento, controle e avaliação.

Na sequência é apresentado o Quadro 1 para ilustrar a evolução do orçamento ao longo dos tempos.

Quadro 1: Evolução dos processos orçamentários

\begin{tabular}{|c|c|c|c|c|c|}
\hline 1900 & 1920 & 1970 & 1970 & 1980 & 2000 \\
\hline $\begin{array}{l}\text { Orçamento } \\
\text { Empresarial }\end{array}$ & $\begin{array}{l}\text { Orçamento } \\
\text { Contínuo }\end{array}$ & $\begin{array}{l}\text { Orçamento } \\
\text { Base Zero }\end{array}$ & $\begin{array}{l}\text { Orçamento } \\
\text { Flexível }\end{array}$ & $\begin{array}{l}\text { Orçamento } \\
\text { Atividades }\end{array}$ & $\begin{array}{l}\text { Beyond } \\
\text { Budgeting }\end{array}$ \\
\hline $\begin{array}{lr}\text { Projeção } & \text { de } \\
\text { recursos } & \\
\text { baseado } & \text { nos } \\
\text { objetivos } & \text { e } \\
\text { controle } & \text { por } \\
\text { meio } & \text { do } \\
\text { acompanhamen } \\
\text { to pelos dados } \\
\text { contábeis }\end{array}$ & $\begin{array}{l}\text { Renovação } \\
\text { do período } \\
\text { concluído e } \\
\text { acréscimo do } \\
\text { mesmo } \\
\text { período no } \\
\text { futuro. }\end{array}$ & $\begin{array}{l}\text { Projeção } \\
\text { dos } \\
\text { recursos da } \\
\text { estaca zero } \\
\text { com } \\
\text { justificativ } \\
\text { a para } \\
\text { todos os } \\
\text { novos } \\
\text { gastos }\end{array}$ & $\begin{array}{l}\text { Projeção } \\
\text { dos recursos } \\
\text { para vários } \\
\text { níveis de } \\
\text { atividades }\end{array}$ & $\begin{array}{l}\text { Projeção dos recursos } \\
\text { nas atividades por meio } \\
\text { de direcionadores. }\end{array}$ & $\begin{array}{l}\text { Projeção dos } \\
\text { recursos de forma } \\
\text { descentralizada e } \\
\text { flexível, guiado } \\
\text { por um conjunto } \\
\text { de princípios. }\end{array}$ \\
\hline
\end{tabular}

Fonte: Lunkes (2007).

Conforme Bornia e Lunkes (2007) na primeira fase do orçamento predominaram o orçamento empresarial com a projeção dos recursos baseado nos objetivos e o controle por meio do acompanhamento dos dados oriundos da contabilidade. A segunda fase orçamentária privilegiou o orçamento contínuo. Este tem como ênfase uma renovação do período concluído e acréscimo do mesmo período no futuro.

A terceira fase foi o orçamento de base zero com a projeção dos recursos da estaca zero com justificativa para todos os gastos novos. A quarta etapa corresponde ao orçamento flexível que destaca a projeção dos recursos para vários níveis de atividade. Posteriormente, surgiu o orçamento por atividades. Este processo orçamentário é uma extensão do custeio baseado por atividades. O Orçamento por atividades apresenta projeção dos recursos nas atividades por meio dos direcionadores. O processo orçamentário mais recentemente elaborado é o Além do Orçamento - Beyond Budgeting. Este utiliza meios mais relativos e adaptáveis de planejamento, avaliação e controle de desempenho. É um modelo de gestão formado por um conjunto de princípios, os quais são guias de atuação empresarial.

Nenhum sistema de orçamento ou de planejamento pode esperar resolver todos os problemas administrativos. Nos orçamentos tradicionais, geralmente não há metas bem definidas e o envolvimento na elaboração do orçamento restringe-se apenas a alta administração, ou seja, não há uma participação efetiva dos funcionários da empresa. Essas projeções são feitas considerando-se os orçamentos dos anos anteriores e isso 
normalmente gera como resultado as mesmas falhas e perpetuação dos erros (Soares, 2011).

O estudo de Sandalgaard e Nikolaj (2014) indica que o Beyond Budgeting não é uma solução padrão, tão pouco se adéqua a todos os tipos de organizações. A organização composta por estrutura cooperativa é um fator que poderá influenciar na implantação do Beyond Budgeting.

Os Princípios de descentralização de poder são: Governança, Desempenho, Empowerment, Responsabilidade, Responsabilidade pelos Clientes, e Informação. No item Governança devem ser esclarecidos e divulgados os princípios e valores da empresa. Os gestores fornecem princípios e limites bem identificados, reúnem as pessoas para um objetivo comum e compartilham valores. No item Desempenho cria-se um clima de alta performance com base no sucesso relativo. No princípio Empowerment dá-se às equipes liberdade, capacidade e autoridade para agir.

No tocante a Responsabilidade, os funcionários empenham-se na busca de resultados competitivos com responsabilidade sobre o desempenho de suas divisões. Quando se refere a Responsabilidade pelos clientes, o foco está no atendimento as necessidades dos clientes, sem pressões para atingir metas, o que melhora o relacionamento a longo prazo. O sexto princípio é a Informação que deve estar disponível de forma ética e transparente para facilitar o aprendizado e encorajar o comportamento ético.

Os princípios de flexibilização de processos são: Metas, Motivação e Recompensa, Planejamento, Recursos, Coordenação, e Mensuração e Controle.

O princípio Metas corresponde a fixação destas com base no benchmark. As equipes devem perseguir um objetivo comum à organização, buscando a excelência dentro e fora da empresa. Deve ser adotada a definição de metas práticas relacionadas com melhorias desconectadas da avaliação de desempenho e premiações. No que se refere a Motivação e Recompensa, estas devem estar alinhadas com o progresso da empresa como um todo.

A empresa passa a recompensar um grande grupo relacionando o desempenho competitivo que a empresa está obtendo. No princípio de Planejamento a estratégia deve estar constantemente alinhada com os processos da empresa. Quanto aos Recursos, estes devem ser liberados de acordo com a necessidade da empresa e agilmente. A utilização de sistemas de previsões dinâmicas pode ser mais eficaz que orçamentos tradicionais.

A Coordenação de todos os planos da organização deve ser feitos de forma dinâmica, por meio de mecanismos de mercado e não de ciclos anuais de planejamento. O sexto princípio é a Mensuração e Controle, onde as informações devem ser claras e concisas e as providências rápidas. Sistemas ERP permitem o gerenciamento das informações e evitam a manipulação de dados.

Ao compreender estes princípios diz-se que a gestão Beyond Budgeting é baseada na descentralização do poder e na flexibilização de processos. A utilização conjunta de diversas ferramentas de gestão em conjunto, tais como Rolling forecast, Benchmarking, Balanced Scorecard, EVA, CRM, completa o modelo Beyond Budgeting. O Beyond Budgeting gera um ambiente de trabalho auto-gerenciado e uma cultura de responsabilidade pessoal, promovendo motivação, produtividade e melhorias nos serviços prestados (Campos \& Krom, 2006).

Quadro 2: Comparativo de elementos na gestão das empresas

\begin{tabular}{lll}
\hline Descrição & Orçamento Tradicional & Beyond Budgeting \\
\hline Metas & As metas são fixadas anualmente & $\begin{array}{l}\text { As metas não são fixas, mas continuamente } \\
\text { monitoradas contra um dado benchmarking, } \\
\text { preferencialmente externo, negociado com o } \\
\text { grupo de gerentes. }\end{array}$ \\
\hline $\begin{array}{l}\text { Premiações } \\
\text { Bonificações }\end{array}$ & e $\begin{array}{l}\text { Os executivos recebem desde que se } \\
\text { enquadrem nos objetivos das metas }\end{array}$ & $\begin{array}{l}\text { Existe a confiança do recebimento da } \\
\text { premiação a partir da avaliação do grupo de } \\
\text { gestores que analisa o desempenho na } \\
\text { abordagem "daquilo que deveria ser feito" }\end{array}$ \\
\hline Planos & $\begin{array}{l}\text { A figura das metas fixas (contratos } \\
\text { fixos) está relacionada aos planos a confiança de que qualquer ação } \\
\text { possa ser exigida para atingir metas de médio } \\
\text { prazo aceitas pelo grupo de gestão, dentro } \\
\text { dos princípios de governança e parâmetros } \\
\text { estratégicos da entidade. }\end{array}$ \\
&
\end{tabular}


Orçamento e estratégia: Beyond Budgeting como ferramenta de planejamento e controle orçamentário em um curso de graduação

\begin{tabular}{|c|c|c|}
\hline Recursos & $\begin{array}{l}\text { Os recursos cuja aceitação de } \\
\text { disponibilidade para apoiar os } \\
\text { orçamentos operacionais e de capital } \\
\text { colocados à disposição. }\end{array}$ & $\begin{array}{l}\text { Existe a confiança de prover os recursos } \\
\text { quando forem necessários. }\end{array}$ \\
\hline Coordenação & $\begin{array}{l}\text { As atividades serão coordenadas com } \\
\text { outros gestores de planos de acordo } \\
\text { com plano aceito ou redirecionado } \\
\text { por níveis superiores. }\end{array}$ & $\begin{array}{l}\text { Existe delegação de confiança para que o } \\
\text { gestor coordene as atividades a partir de } \\
\text { acordos periódicos e exigências de clientes }\end{array}$ \\
\hline Controles & $\begin{array}{l}\text { O desempenho deve ser monitorado } \\
\text { mensalmente e qualquer variação } \\
\text { significativa deve ser revisada. } \\
\text { Forecast } \\
\text { trimestralmente. }\end{array}$ & $\begin{array}{l}\text { Existe confiança de que o forecast seja } \\
\text { baseado na alternativa mais provável, de } \\
\text { maneira que só existirá interferência se a } \\
\text { tendência de os indicadores ficar fora de } \\
\text { certos parâmetros. }\end{array}$ \\
\hline
\end{tabular}

Fonte: Frezatti (2005, p.30).

Os orçamentos são comumente vistos como um componente central dos sistemas de controle de gerenciamento. A literatura sobre Beyond Budgeting argumenta que os gerentes podem desenvolver outros controles para substituir os orçamentos (O'Grady \& Akroyd, 2016).

Relativas a abordagens de orçamento, foram identificadas duas tendências. $\mathrm{Na}$ primeira abordagem (Beyond Budgeting), o desacoplamento da fixação de metas e previsão era a questão chave, enquanto que na outra prática (o orçamento anual simplificado), a questão da dissociação permaneceu ambíguo e, além disso, manteve várias características de orçamento tradicional (Henttu-Aho \& Järvinen, 2012).

Os programas de educação à distância possuem uma reputação de serem mais efetivos quando se trata de custos, do que os sistemas tradicionais de ensino. Mas diversos estudos têm mostrado que se torna realidade apenas em casos onde as matrículas conseguem alcançar níveis elevados quando comparados aos gastos e às taxas de conclusão. Desta forma, o Skandia Navigator por meio de seus drivers de mudança institucional é uma ferramenta que pode auxiliar no planejamento e na mensuração dos resultados organizacionais (Soares \& Mazon, 2016).

\section{MÉTODO}

Optou-se nesta pesquisa pela metodologia qualitativa de estudo de caso, verificando como os fatores de um fenômeno influenciou a relação entre orçamento e estratégia no caso estudado. Em se tratando de uma pesquisa qualitativa, o estudo de caso permitiu aos pesquisadores direcionar especificamente 0 fenômeno pesquisado possibilitando a profundidade do estudo, identificando informações que permitam ao desenvolvimento da relação orçamento e estratégia.

Na pesquisa qualitativa, o caso a ser analisado é definido intencionalmente para que se entenda um fenômeno específico, um "revelatory case". Esta situação ocorre quando o pesquisador tem a oportunidade de observar e analisar uma situação científica previamente não acessível.

Devido às singularidades deste trabalho, utilizouse o método do estudo de caso, no qual a unidade social investigada - curso de graduação a distância foi intencionalmente escolhida e analisada. O sujeito da pesquisa foi o coordenador do curso analisado.

Quanto à pesquisa de campo, a coleta de dados foi realizada por meio de entrevista semi-estruturada junto ao coordenador do curso e por pesquisa documental em documentos institucionais, por considerar que com esta técnica é possível construir informações pertinentes para o cumprimento do objetivo desta pesquisa.

$\mathrm{Na}$ sequência é apresentado um quadro que direciona como a coleta de dados ocorreu e como ela contribui para o desenvolvimento da pesquisa. 
Quadro 3 - Coleta de dados

Coleta de dados
Plano de Desenvolvimento Institucional

Projeto Pedagógico do Curso

Plano Anual de Trabalho

Entrevista com o coordenador

Fonte: Elaborado pelos autores, 2018.

A análise dos dados se inicia com a transcrição da entrevista. Com a transcrição foi possível categorizar as ações nos doze princípios do Beyond Budgeting. Entendendo o posicionamento estratégico da empresa e com as informações da pesquisa documental e entrevista, pode-se elaborar um orçamento capaz de apresentar informações da operação da universidade.

\section{APRESENTAÇÃO DOS RESULTADOS}

Para a análise da estratégia adotada pela universidade, começou-se verificando a missão da universidade, conforme o PDI (2018, p.18)

"...é uma Universidade Comunitária com a missão de promover educação, em todos os níveis e modalidades, para formar integralmente e ao longo da vida, cidadãos competentes, comprometidos com o desenvolvimento da ciência, da tecnologia e da inovação, contribuindo para a melhoria da vida em sociedade."

No PPC (2014, p.31) do curso observou-se uma característica importante do sistema tutorial:

“... atividades docentes são realizadas a partir da atuação de quatro agentes principais: o professor conteudista, o coordenador do curso, o professor da turma e o tutor. O Professor da turma é o responsável principal pelo desenvolvimento do processo de ensino e aprendizagem. É o especialista nos conteúdos e estratégias que compõe a unidade de aprendizagem, com quem o estudante conta permanentemente."

Outra característica identificada na entrevista com o coordenador do curso está na adoção de professores com titulação de mestrado e doutorado para serem tutores em disciplinas.

Para operacionalizar a estratégia, optou-se pela elaboração do Beyond Budgeting. Este processo é
Informações coletadas

Histórico da IES, Inserção Social, Missão, Visão e Valores, Metas Institucionais, Projeto Pedagógico Institucional, Organização Didático Pedagógica, ou seja, um conjunto de informações para definir o posicionamento estratégico da universidade.

Objetivos do Curso, Organização Curricular,
Organização Didático Pedagógica, Metodologia para Modalidade a Distância, Corpo Docente, Ações de investimento e rotinas do curso. Ações de investimento e rotinas do curso, informações acerca de valores.

baseado em doze princípios, sendo seis focados na descentralização do poder nas empresas e os outros seis na flexibilização de processos.

Os Princípios de descentralização de poder são: Governança, Desempenho, Empowerment, Responsabilidade, Responsabilidade pelos Clientes, e Informação.

- Governança: todos os professores que irão atuar no curso passam por uma capacitação quanto a metodologia da instituição e como proceder enquanto professor a distância. Este curso tem valor estimado em $\mathrm{R} \$ 280$ por professor e duram dois meses entre atividades presenciais e atividades a distância.

- Desempenho:os professores são acompanhados quantitativa e qualitativamente de forma periódica. $\mathrm{Na}$ instituição existe um setor responsável pelo acompanhamento docente. Assim, o professor recebe feedback sobre o seu desempenho no espaço virtual de aprendizagem. No tocante ao desempenho quantitativo o professor tem um dia para responder as perguntas dos alunos, três dias para corrigir a avaliação a distância e três dias para publicar mural.

Já na parte qualitativa é avaliado o feedback do professor quanto a avaliação, a utilização do mural e da ferramenta professor. Na resposta acerca da avaliação do aluno tem-se: cordialidade, evidência de acertos, identificação dos erros, motivação aos alunos, e promove reflexão sobre os erros. Quanto a utilização do mural é analisado se este foi utilizado de forma informativa, motivacional, e orientadora, bem como se a linguagem foi cordial, criativa e objetiva. No tocante a ferramenta professor é analisada a função motivacional e orientadora das respostas dadas aos alunos bem como se a linguagem foi cordial e objetiva. 

e controle orçamentário em um curso de graduação

- Empowerment: o professor é o responsável pelo desenvolvimento das atividades. $\mathrm{O}$ professor produz a avaliação presencial e a distância, tem liberdade para colocar materiais extras para leituras dos alunos, bem como propor fóruns para debates, gravar e inserir vídeos.

-Responsabilidade: o professor ao participar do programa sabe quais são os indicadores de desempenho que ele será avaliado. O professor recebe feedback sobre o seu desempenho no espaço virtual de aprendizagem, desta forma empenham-se na busca de resultados competitivos com responsabilidade sobre o desempenho de suas ações.

-Responsabilidade pelos clientes: o foco dos cursos está no atendimento as necessidades dos alunos, procurando desenvolver materiais online de qualidade, com uma ergonomia no espaço virtual de aprendizagem. Não existe uma quantidade mínima de alunos para iniciar as turmas.

- Informação: o professor conhece quais são os indicadores e o que precisa fazer para ter um desempenho aceitável.

Os princípios de flexibilização de processos são: Metas, Motivação e Recompensa, Planejamento, Recursos, Coordenação, e Mensuração e Controle.

-Metas: as metas para o ano de 2016 foram: ajustar o espaço virtual de aprendizagem de acordo com as sugestões viáveis dos alunos e professores nos relatórios de 2015; realizar ajuste no material didático de cinco disciplinas que necessitavam atualização; capacitar os dezoito professores que lecionarão as disciplinas. As metas não são fixas, mas continuamente monitoradas e negociadas com o grupo de gerentes (coordenação do curso, setor de produção de material e direção financeira).

- Motivação e Recompensa: a remuneração do professor deriva de sua titulação. Existe a confiança do recebimento da premiação a partir da avaliação que analisa o desempenho na abordagem "daquilo que deveria ser feito", pois o professor com bom desempenho poderá ser realocado permanentemente no curso.

- Planejamento: existe confiança de que o forecast seja baseado na alternativa mais provável. A estratégia deve estar constantemente alinhada com os processos da empresa de ofertar educação a distância com qualidade. Assim são elaborados orçamentos e avaliações de desempenho. Estas atividades são feitas por um conjunto de setores, a coordenação do programa, o setor financeiro da instituição, o setor de produção de material didático e o setor de apoio aos professores.

- Recursos: os pagamentos são realizados mensalmente. A previsão de gastos é feita a cada semestre onde ocorre o ingresso de novos alunos e egresso de formandos.

- Coordenação: os planos da organização são feitos por meio de mecanismos de mercado. Os dados como projeção de número de alunos, turmas, professores, novos materiais didáticos, ajustes no material didático, capacitação para professores e novos alunos, bem como gastos administrativos são considerados. Existe delegação de confiança para que o coordenador do curso coordene as atividades a partir de acordos periódicos e exigências de clientes.

- Mensuração e Controle: existe a mensuração contábil dos gastos e receitas do curso, bem como uma mensuração pedagógica (descrita no item desempenho). Ao final de cada disciplina é feita uma avaliação da disciplina e do curso, por parte dos alunos. Esta avaliação é por meio de um questionário com Escala Likert e está disponível no espaço virtual. Este questionário faz parte do programa de avaliação interna da universidade.

Assim, como base nas informações descritas até então se pode elaborar o conjunto de informações orçamentárias para o ano de 2016 conforme a seguir, se baseando nos princípios do Beyond Budgeting.

A receita de vendas líquida orçada é de $R \$$ $305.223,60$, pois a projeção é que existam 88 alunos pagando mensalidade de $\mathrm{R} \$ 248,00$, sendo que 25 possuirão desconto por conta de algum convênio da instituição. Assim a receita bruta mensal é de $\mathrm{R} \$$ 26.774,00, o que acarretará em uma receita anual de $\mathrm{R} \$ 305.223,60$, reduzindo o valor histórico de inadimplência do curso $(5 \%)$ chega-se a receita líquida de $\mathrm{R} \$ 25.435,30$.

O quadro 4 apresenta o orçamento de vendas desenvolvido por meio de mecanismos de mercado e das informações do planejamento para o programa para o ano de 2016. 
Quadro 4: Orçamento de vendas

\begin{tabular}{ll}
\hline Orçamento de Vendas & Curso \\
\hline Número de Alunos Regulares & 88 \\
\hline Valor da Mensalidade & $\mathrm{R} \$ 248,00$ \\
\hline Receita com Alunos Regulares & $\mathrm{R} \$ 21.824,00$ \\
\hline Número de Alunos com Desconto & 25 \\
\hline Valor da Mensalidade com Desconto & $\mathrm{R} \$ 198,00$ \\
\hline Receita com Alunos com Desconto & $\mathrm{R} \$ 4.950,00$ \\
\hline Inadimplência & $\mathrm{R} \$ 1.338,70$ \\
\hline Receita Mensal & $\mathrm{R} \$ 25.435,30$ \\
\hline Receita Anual & $\mathbf{R} \$ \mathbf{3 0 5 . 2 2 3 , 6 0}$
\end{tabular}

Fonte: Elaborado pelo autor, 2018.

Os gastos diretos são orçados dentro de seis grandes grupos: Despesas com o desenvolvimento de competências/professor; Despesas com salários dos professores; Despesas com o desenvolvimento de material; Despesas com adaptação de material; Despesas com coordenação de curso; Despesas com palestras/aula inaugural.

$\mathrm{O}$ valor total de gastos diretos foi orçado em $\mathrm{R} \$$ $\$ 202.458,80$. As despesas com o desenvolvimento de competências/professor correspondem a R $\$ 5.040,00$, pois serão capacitados dentro da metodologia $\mathrm{EaD}$ da universidade 18 professores com um valor de $\mathrm{R} \$ 280,00$ cada. Despesas com salário dos professores corresponderá a $\mathrm{R} \$ 102.960,00$, pois serão 52 turmas de 60 horas com valor da hora médio de $\mathrm{R} \$ 33,00$.

Como o pagamento será feito na folha de pagamento do funcionário ainda serão acrescidos décimo terceiro e férias proporcionais além dos encargos sociais, o que corresponde a um total de $\mathrm{R} \$ 154.668,80$.

Quadro 5: Orçamento de gastos diretos

\begin{tabular}{ll}
\hline Orçamento de gastos diretos & Curso \\
\hline Número de turmas & 52 \\
\hline Número de professores & 18 \\
\hline Número de materiais novos & 0 \\
\hline Número de materiais adaptados & 5 \\
\hline Capacitação por professor & $\mathrm{R} \$ 280,00$ \\
\hline Capacitação dos professores & $\mathrm{R} \$ 5.040,00$ \\
\hline Salário por turma & $\mathrm{R} \$ 1.980,00$ \\
\hline Salário total & $\mathrm{R} \$ 102.960,00$ \\
\hline 13o. e férias proporcional & $\mathrm{R} \$ 11.440,00$ \\
\hline Encargos & $\mathrm{R} \$ 40.268,80$ \\
\hline Custo total com professores & $\mathrm{R} \$ 154.668,80$ \\
\hline Valor de produção de material & $\mathrm{R} \$ 2.320,00$ \\
\hline
\end{tabular}


Orçamento e estratégia: Beyond Budgeting como ferramenta de planejamento e controle orçamentário em um curso de graduação

\begin{tabular}{ll}
\hline Valor total de produção de material & $\mathrm{R} \$ 0,00$ \\
\hline Valor de adaptação de material & $\mathrm{R} \$ 770,00$ \\
\hline Valor total de adaptação de material & $\mathrm{R} \$ 3.850,00$ \\
\hline Coordenação & $\mathrm{R} \$ 36.400,00$ \\
\hline Palestra & $\mathrm{R} \$ 2.500,00$ \\
\hline Gastos Diretos Totais & $\mathbf{R} \$ \mathbf{2 0 2 . 4 5 8 , 8 0}$
\end{tabular}

Fonte: Elaborado pelo autor. 2018

Os gastos administrativos podem ser agrupados em seis categorias: Investimento em Tecnologia da Informação (TI); Investimento no suporte aos alunos; Investimento no treinamento de alunos; Investimento no suporte ao professor; Gasto com setor de desenvolvimento de material/acompanhamento pedagógico; e Rateio de demais gastos fixos.

$\mathrm{O}$ investimento em TI está orçado em $\mathrm{R} \$ 5.256,55$. Na universidade existe um setor que auxilia no suporte ao sistema de aprendizagem, suporte operacional da rede e investimento na rede. $\mathrm{O}$ valor rateado pela universidade e identificado ao curso é de $\mathrm{R} \$ 52.356,55$.

O investimento no suporte aos alunos é de $\mathrm{R} \$ 3.414,12$. Na universidade existem dois setores de apoio aos alunos, um acadêmico e outro motivacional. Um setor é responsável pelo suporte financeiro, de matrícula etc. Outro de acesso ao espaço virtual, aviso aos alunos, contato telefônico etc.

O investimento no treinamento de alunos está orçado em $\mathrm{R} \$ 1.760,00$. Cada um dos 88 alunos do programa antes de iniciar o curso participa de uma atividade de conhecimento da metodologia e espaço que ele utilizará durante o curso. $\mathrm{O}$ custo por aluno desta atividade é de $\mathrm{R} \$ 20,00$, totalizando $\mathrm{R} \$ 1.760,00$.

O investimento no suporte ao professor está orçado em $\mathrm{R} \$ 2.773,14$. Na universidade existe um setor responsável pelo monitoramento da atividade do professor e pela avaliação de desempenho deste.

$\mathrm{O}$ gasto com setor de desenvolvimento de material ficou estipulado em $\mathrm{R} \$ 7.289,97$. Este setor serve de apoio durante a produção do material desenvolvido pelos professores.

Todos os demais gastos fixos da instituição estão orçados em Rateio de demais gastos fixos: $\$ 17.798 .56$.

O quadro 6 apresenta o orçamento de gastos administrativos, detalhado para o curso, desenvolvido por meio das informações do planejamento para o programa para o ano de 2016.

Quadro 6: Orçamento de gastos administrativos

Orçamento de gastos administrativos

Curso

\begin{tabular}{ll}
\hline Alunos & 88 \\
\hline Valor do Treinamento & $\mathrm{R} \$ 20,00$ \\
\hline Treinamento de Alunos & $\mathrm{R} \$ 1.760,00$ \\
\hline Investimento em TI & $\mathrm{R} \$ 5.256,55$ \\
\hline Suporte aos Alunos & $\mathrm{R} \$ 3.414,12$ \\
\hline Suporte ao Professor & $\mathrm{R} \$ 2.773,14$ \\
\hline Desenvolvimento de Material & $\mathrm{R} \$ 7.289,97$ \\
\hline Demais Gastos Fixos & $\mathrm{R} \$ 17.798,56$ \\
\hline Gastos Administrativos Totais & $\mathbf{R} \mathbf{3 8 . 2 9 2 , 3 4}$ \\
\hline
\end{tabular}

Fonte: Elaborado pelos autores, 2018

Assim, resumidamente as informações - Despesas com o desenvolvimento de material: $\$ 0$ orçamentárias são as seguintes: • Despesas com adaptação de material: $\$ 3.850,00$

- Receita Operacional Líquida: R \$ 305.223,60 - Despesas com coordenação de curso: \$36.400,00

- Total de Gastos Diretos: \$202.458,80

- Despesas com o desenvolvimento de • Total de Gastos Administrativos: R $\$ 38.292,34$ competências/professor: $\$ 5.040,00$

- Despesas salário dos professores: $\$ 154.668,80$

- Despesas com palestra/aulas inaugural: $\$ 2.500,00$

- Investimento em TI: R \$ 5.256,55

- Investimento no suporte aos alunos: $\mathrm{R} \$ 3.414,12$ 
- Investimento no treinamento de alunos: $\mathrm{R} \$$ $1.760,00$

- Investimento no suporte ao professor: $\mathrm{R} \$ 2.773,14$

- Gasto com setor de desenvolvimento de material: $\mathrm{R} \$ 7.289,97$

- Rateio de demais gastos fixos: R $\$ 17.798,56$

\section{DISCUSSÃO DOS RESULTADOS}

O conjunto de informações destacados na apresentação dos dados caracteriza o posicionamento estratégico de Diferenciação de Porter (2004), pois com a diferenciação se oferece e serviços com valor agregado, considerado diferenciado quando comparado com os concorrentes. A universidade em seus cursos a distância possui professores titulares atuando no papel de tutor dos cursos.

Pode-se identificar também nos conceitos de Serra, Torres e Torres (2004), o componente posicionamento da estratégia, pois se criou uma posição valiosa e única envolvendo um conjunto de atividades, assim, desempenhando as mesmas atividades (de tutoria) de diferentes maneiras das exercidas pelos competidores (com a utilização de professores, os mesmo que desenvolvem os materiais didáticos).

Com a elaboração do orçamento para o curso de graduação foi possível verificar a relação entre o orçamento e estratégia organizacional, corroborando assim os estudos de Simons (1987); Langfield-Smith, (1997); Guilding, Cravens e Tayles, (2000); Hansen e Van der Stede (2004); Backes, et al (2004); Almeida, et al (2009); Neitzke e Espejo (2016); Silva e Rissi (2017) e Melo et al (2017). No caso estudado observou-se a estratégia de Diferenciação de Porter (2004)

Souza e Lavarda (2011, p.1) concluem que para os gestores que escolhem o Beyond Budgeting não percebem grandes mudanças em suas instituições, já que a maior parte dos benefícios propostos pelo Beyond Budgeting já são utilizadas nas instituições com o uso do modelo orçamentário tradicional, ou seja, as críticas a esse modelo não se aplicam às IES observadas.

Os resultados apresentados neste estudo de caso confrontam o que foi apresentado por Souza e Lavarda (2011). Isto porque a utilização do Beyond Budgeting permitiu resolver as três das principais críticas que são normalmente apontadas ao processo tradicional de elaboração dos orçamentos: 1) metas inflexíveis, 2) modelo orçamentário constitui uma barreira a mudanças, principalmente no que diz respeito à alocação de recursos e 3) na elaboração do orçamento, apenas a alta administração participa, não dando espaço para quem realmente "faz acontecer".

As vantagens obtidas na utilização do Beyond Budgeting, para o caso específico da educação à distância:

1) As metas são mais flexíveis: as metas não são fixas, mas continuamente monitoradas e negociado com o grupo de gerentes (coordenação do programa de cursos, setor de produção de material e direção financeira);

2) Menor resistência a mudanças: o professor recebe feedback sobre o seu desempenho no espaço virtual de aprendizagem. Existe uma avaliação de desempenho quantitativa e outra qualitativa, assim está apto para mudanças;

3) Mais participativa: os planos da organização são feitos por meio de mecanismos de mercado. Os dados como projeção de número de alunos, turmas, professores, novos materiais didáticos, ajustes no material didático, capacitação para professores e alunos, bem como gastos administrativos são considerados. Existe delegação de confiança para que o coordenador do programa coordene as atividades, junto aos professores e direção de campus, a partir de acordos periódicos e exigências de clientes.

O cálculo das receitas e dos custos para os diferentes cursos de educação à distância, efetuados pelo Beyond Budgeting, são mais confiáveis dos que os que seriam utilizados pelo método tradicional, isto porque existe confiança de que o forecast é baseado na alternativa mais provável. A estratégia deve estar constantemente alinhada com os processos da empresa de ofertar educação a distância com qualidade. Assim são elaborados orçamentos e avaliações de desempenho. Estas atividades são feitas por um conjunto de setores, a coordenação do programa, o setor financeiro da instituição, o setor de produção de material didático e o setor de apoio aos professores.

\section{CONCLUSÃO}

O objetivo deste artigo consistia analisar o posicionamento estratégico de um curso de graduação de ensino a distância e verificar a sua operacionalização por meio do orçamento. Após o exposto na análise dos dados considera-se que o objetivo do artigo foi alcançado.

Primeiramente identificou-se a tipologia de Porter (2004), concernente a estratégia diferenciação 
Orçamento e estratégia: Beyond Budgeting como ferramenta de planejamento e controle orçamentário em um curso de graduação

do produto, foi utilizada pela universidade como estratégia organizacional.

A operacionalização da estratégia por meio do Beyond Budgeting foi utilizada para evidenciar a relação entre o posicionamento estratégico do curso, o planejamento estratégico e orçamento.

Quanto à natureza do orçamento como uma prática institucionalizada, a literatura aborda duas questões principais: como os processos orçamentais das organizações refletem o ambiente institucional de fora, bem como a natureza das rotinas orçamentárias como prática altamente institucionalizada (Henttu-Aho \& Järvinen, 2012). A adoção do Beyond Budgeting permitiu que a instituição observasse o ambiente externo, ou seja, tendo o seu planejamento orçamentário baseado na concorrência ou a satisfação dos clientes.

Beyond Budgeting corresponde a descentralização do poder e na flexibilização de processos na elaboração do orçamento. A elaboração do Beyond Budgeting apresentados nesta pesquisa seguiu os princípios de descentralização de poder que foram: governança, desempenho, empowerment, responsabilidade, responsabilidade pelos clientes, e informação. Os princípios de flexibilização de processos foram: metas, motivação e recompensa, planejamento, recursos, coordenação, e mensuração e controle.

Os programas de educação à distância possuem uma reputação de serem mais efetivos quando se trata de custos, do que os sistemas tradicionais de ensino. Mas diversos estudos têm mostrado que se torna realidade apenas em casos onde as matrículas conseguem alcançar níveis elevados quando comparados aos gastos e às taxas de conclusão. Desta forma o Beyond Budgeting é uma ferramenta que pode ajudar no planejamento. Isto porque as vantagens obtidas na utilização do Beyond Budgeting, para o caso específico da educação à distância, são três: as metas são mais flexíveis; menor resistência a mudanças; e mais participativa.

O tema apresenta relevância por relacionar a diferenciação estratégica de Porter com o orçamento. Por meio da operacionalização do Beyond Budgeting pode-se concretizar o planejamento orçamentário de um curso com estratégia de diferenciação. Esse modelo gerará gestão inovadora, que no longo prazo poderá aportar vantagem competitiva para as organizações.

Espera-se que os resultados deste artigo sirvam como ponto inicial para o desenvolvimento de outras pesquisas, tais como a aplicação em outros serviços. $\mathrm{E}$, por fim, acredita-se que, com o aprofundamento dos estudos, será possível retroalimentar o processo de desenvolvimento científico.

\section{REFERÊNCIAS}

Almeida, L. B; Machado, E. A.; Raifur, L. \& Nogueira, D. R. (2009). A Utilização do orçamento como ferramenta de apoio à formulação de estratégia, de controle e de interatividade: um estudo exploratório nas cooperativas agropecuárias da região sul do Brasil. Contabilidade Vista \& Revista, 20: 65-99.

Backes, B I; Silva, S C, Daga, S M \& Selig, P M. (2004). A vinculação do orçamento à estratégia para garantir a eficácia do planejamento e controle dos fatores críticos de sucesso da cadeia de logística. XI Congresso Brasileiro de Custos, Porto Seguro, Brasil.

Berland, N.; Curtis, E. \& Sponem, S. (2018). Exposing organizational tensions with a nonTraditional budgeting system.Qualitative Research in Accounting \&Management , 19 .

Bornia, A. C. \& Lunkes, R. J.(2007).Uma contribuição à melhoria do processo orçamentário. Contabilidade Vista \& Revista, 18:.37-59.

Campos, J. C. De \& Krom, V. (2006).Beyond budgeting: uma alternativa de gestão financeira além dos orçamentos. Artigo apresentado no VI Encontro Latino Americano de Pós-Graduação, Vale do Paraíba,Brasil.

Frezatti, F. (2005). Beyond budgeting: Inovação ou resgate de antigos conceitos do orçamento empresarial? RAE - Revista de Administração de Empresas, 45: 23-33.

Guilding, C.; Cravens, K. S. \& Tayles, M. (2015). An international comparison of strategic management accounting practices. Management Accounting Research, 11: 113- 135.

Hansen, S. C.\& Van DerStede, W. (2004). Multiple facets of budgeting: an exploratory analysis. Management Accounting Research, 15: 415-439.

Henttu-Aho, T. \& Järvinen, J. (2012). A field study of the emerging practice of beyond budgeting in industrial companies: an institutional perspective. European Accounting Review, 17. 
Langfield-Smith, K (1997). Management control systems and strategy: a critical review. Accounting, Organizations and Society, 22: 207-232.

Lunkes, R. J.(2007). Manual de orçamento.São Paulo: Atlas, Brasil.

Maciel, C. O. (2007). Condições para a formação da intenção estratégica: isolando seus efeitos na variação do desempenho de pequenas empresas. Perspec. Contemp., 2: 120-143.

Melo,H P; Silva G R; Nicolau, A M \& Lima, A C. (2017). O uso do orçamento como instrumento de gestão à formulação de estratégia e controle: um estudo de caso em empresa do setor agroindustrial da Paraíba Revista Ambiente Contábil,. 9: 290 - 305.

Neitzke, A. C. A. \& Espejo, M. M. dos S. B. (2016). Estratégia e seus impactos no orçamento empresarial: um estudo contributivo à tipologia de Porter e ao modelo de Simons. Brazilian Journal of Quantitative Methods Applied to Accounting, 3: 119.

O'Grady, W. \& Akroyd, C. (2016).The MCS package in a non-budgeting organisation: a case study of Mainfreight.QualitativeResearch in Accounting\&Management, 13: 2-30.

Porter, M.E. (2004). Estratégia competitiva: técnicas para análise de indústrias e da concorrência. Rio de Janeiro: Campus.
Sandalgaard, N. \& NikolajBukh, P. (2014).Beyond Budgeting and change: a case study. Journal of Accounting \& Organizational Change, 10:409-423.

Silva, M W \& Rissi, M. (2017). Orçamento e estratégia na gestão universitária: um estudo bibliométrico. XVII Colóquio de Gestão Universitária, Mar Del Plata, Argentina.

Simons, R. (1987). Accounting control systems and business strategy: an empirical analysis. Accounting, Organizations and Society, v: 357-374.

Serra, F. A. R, Torres, M. C. S., Torres, A.P.. (2004) Administração Estratégica: Conceitos, Roteiro Prático e Casos. Rio de Janeiro: Reichmann \& Affonso Editores.

Soares, T. C.(2011).Custeio baseado em atividades e orçamento baseado em Atividades em um caso industrial. Revista Gestão Industrial, 07:90102.

Soares, T. C.\& Mazon, G. (2016): Autoavaliação e seus Drivers de Mudança Institucional Baseado na Capacidade Absortiva. Revista Ibero-Americana de Estratégia 15: 77-90.

Souza,R. P. S. De \& Lavarda, C. E. F.(2011).Avaliação do Beyond Budgeting como ferramenta de planejamento e controle na visão dos gestores de IES. Revista Contemporânea de Economia e Gestão. 9. 